\title{
Comparative Study of Efficacy and Safety of Microneedling with Topical Autologous Platelet Rich Plasma Versus Microneedling Alone in Facial Atrophic Acne Scars
}

\author{
Pdiangty Giri Mawlong ${ }^{1}$, , Surabhi Dayal ${ }^{2}$, Nishant Bisht ${ }^{3}$ \\ ${ }^{1}$ Dermatology Department, Government Medical College, Haldwani, India \\ ${ }^{2}$ Dermatology Department, Pandit Bhagwat Dayal Sharma Post Graduate Institute of Medical Sciences, Rohtak, India \\ ${ }^{3}$ Surgery Department, Government Medical College, Haldwani, India \\ Email address: \\ pdiangtygmawlong29@gmail.com (P. G. Mawlong) \\ ${ }^{*}$ Corresponding author
}

\section{To cite this article:}

Pdiangty Giri Mawlong, Surabhi Dayal, Nishant Bisht. Comparative Study of Efficacy and Safety of Microneedling with Topical Autologous Platelet Rich Plasma Versus Microneedling Alone in Facial Atrophic Acne Scars. International Journal of Clinical Dermatology.

Vol. 4, No. 2, 2021, pp. 10-15. doi: 10.11648/j.jicd.20210402.11

Received: August 25, 2021; Accepted: September 8, 2021; Published: September 14, 2021

\begin{abstract}
Introduction: Atrophic acne scars are dermal depressions resulting from destruction of collagen after inflammatory acne which can lead to poor self-image and treating them can help improve patients' self-esteem. Objectives: This study compared the efficacy and safety of combination of microneedling using dermaroller with topical application of autologous platelet rich plasma versus microneedling using dermaroller alone in facial atrophic acne scars. Methods: Patients with atrophic acne scars were randomized into 2 groups of 20 patients each and treated with either dermaroller alone or with a combination of dermaroller and topical application of autologous PRP at 4 weeks intervals for 16 weeks. Final response was evaluated at 4 weeks after the last sitting. Goodman and Baron's qualitative acne scarring grading system, Goodman and Baron's quantitative acne scar grading system, physician's global assessment (PGA) and visual analogue scale were used to evaluate the treatment outcome in both the groups. Side effects were also noted at each visit. Results: At the end of treatment, improvement in Goodman and Baron's qualitative acne scar grades, decrease in mean Goodman and Baron's quantitative acne scar scores, percentage decrease in mean Goodman and Baron's quantitative acne scar scores, physician global assessment and visual analogue scale were significantly higher in patients treated with dermaroller in combination with topical autologous PRP as compared to patients treated with dermaroller alone without much adverse effects. Conclusion: The combination approach using dermaroller and PRP was a better treatment option compared to dermaroller alone in atrophic acne scars with similar safety profile.
\end{abstract}

Keywords: Acne Scars, Dermaroller, Platelet Rich Plasma

\section{Introduction}

Acne vulgaris is a chronic pilosebaceous follicle disorder affecting over $90 \%$ of adolescents and continue till adulthood in approximately $12-14 \%$ of cases $[1,2]$. In some, the severe inflammatory response results in textural change in superficial and deep dermis, leading to post acne scars [3]. There are various types of atrophic acne scars, and the classification as proposed by Jacob CI et al is widely accepted. The three types of atrophic acne scars are ice-pick, rolling, and boxcar types [4]. Various treatment modalities are available to treat atrophic acne scars such as fillers, subcission, lasers, chemical peels, dermabrasion, TCA CROSS, microneedling and radiofrequency [5]. Microneedling utilizes tiny needles to puncture the skin multiple times, creating microclefts that penetrate into the dermis. This leads to wound healing and release of growth factors, leading to collagen production and deposition in the upper dermis [1]. Microneedling with dermaroller is a new treatment modality for the treatment of acne scars [6]. The 
classic dermaroller used for acne scars is usually $0.5-3 \mathrm{~mm}$ in length and 0.1-0.25 $\mathrm{mm}$ in diameter [7]. Microneedling triggers the formation of new collagen (natural collagen) and elastin in the papillary dermis without injuring the epidermis $[4,6]$. Platelet rich plasma (PRP) is an autologous solution of plasma which have approximately 4 -7 times the baseline concentration of human platelets which is prepared from centrifuging patients own blood and has been utilized for various dermatological conditions [8]. Growth factors derived from platelets helps in cellular growth, maturation and differentiation and can be utilized to quicken the healing process [9]. PRP has regenerative effect on keratinocytes, endothelial cells, erythrocytes, fibroblasts and collagen [10]. Microneedling creates a clear pathway for PRP to be absorbed more effectively. PRP induced growth factors along with growth factors induced by skin needling act synergistically to enhance the wound healing response [11]. Though there are studies available establishing the role of microneedling and platelet rich plasma in atrophic acne scars but there are few Indian studies comparing the clinical efficacy and safety of combination of microneedling with topical autologous platelet rich plasma versus microneedling alone in atrophic acne scars. This prompted us to undertake this study comparing the efficacy and safety of microneedling using dermaroller versus combination of microneedling using dermaroller with topical application of autologous platelet rich plasma in the treatment of facial atrophic acne scars.

\section{Materials and Methods}

\subsection{Study Design}

The study was a 16-weeks, prospective, randomised interindividual study performed in the department of dermatology, Pt. B.D. Sharma, Rohtak. The study was approved and ethically justified by the ethics committee of Post Graduate Board of Medicine and Allied Sciences, Pt. B.D Sharma, University of Health Sciences, Rohtak (approval letter no. IEC/Th/17/SVD/01).

\subsection{Patient Selection}

Forty patients of atrophic acne scars of age more than 18 years having facial atrophic acne scars grade 2 to grade 4 were included in the study. Acne scars grading was performed using Goodman and Baron's qualitative acne scar grading system [12] which classify acne scars lesions into 4 grades.

Grade 1 acne scar: Grade 1 disease is macular disease, and this may be erythematous or hyper or hypo pigmented, visible to the patient irrespective of distance.

Grade 2 acne scar: Grade 2 disease comprises mildly atrophic or hypertrophic disease that may not be visible from a social distance of $50 \mathrm{~cm}$ or more, is covered adequately by cosmetic makeup or in men the shadow of shaved facial hair.

Grade 3 acne scar: Grade 3 disease denotes moderate acne scarring with significant contour abnormality. This scar is visible at social distances of $50 \mathrm{~cm}$ or greater and is not easily covered by makeup or the shadow of shaved beard hair.

Grade 4 acne scar: Grade 4 disease represents the most severe scarring which is visible at social distances greater than $50 \mathrm{~cm}$, is not covered easily by makeup or the shadow of shaved beard hair in males.

Exclusion criteria include patients with active acne, active herpes labialis, facial warts or molluscum contagiosum, who were on systemic retinoids in the previous 6 months and any anti-acne therapy, oral or topical for last 4 weeks, history of or evidence of keloid scars, pregnant or lactating women, history of any facial surgery or procedure for scars, photosensitivity, active dermatosis, hypersensitivity to formulation used in the study, bleeding disorders or on anticoagulant medications (aspirin, warfarin, heparin), corticosteroid therapy, severe systemic illness or malignancy. Patient with history of diabetes, collagen vascular disease, skin cancer, solar keratosis and patients having unrealistic expectations. A detailed history and clinical examination of all the patients were carried out to rule out all exclusion criteria. Prior written consent was taken from all the patients after explanation of the procedure and baseline complete hemogram were done.

\subsection{Treatment Protocol}

Forty patients were taken for the study and were randomly divided into 2 groups of 20 patients each using a computer-generated randomization chart. Group 1 was treated with microneedling using dermaroller alone while Group 2 was subjected to a combination therapy of microneedling using dermaroller and topical autologous PRP. After gentle cleansing, the skin of the face was anesthetized using topical anesthetic cream under occlusion for about one hour. Skin needling was carried out using a dermaroller studded with 192 microneedles in eight rows and $1.5 \mathrm{~mm}$ in length. The dermaroller was applied sequentially in horizontal, vertical, and diagonal directions till pinpoint bleeding occurred. For the preparation of PRP, a volume of $10 \mathrm{ml}$ of whole blood was collected from the patient and separated into two sterile conical test tubes of $5 \mathrm{ml}$ each containing acidcitrate dextrose. PRP was prepared by using double spin method (low spin followed by high spin method). The PRP was then mixed with $10 \%$ calcium chloride (in the ratio of $1: 10)$ which acts as a platelet activator. After gentle cleansing of the face with antiseptic, microneedling was performed with dermaroller which was applied sequentially in horizontal, vertical, and diagonal directions till pinpoint bleeding occurred followed by 0.2 to $0.3 \mathrm{cc}$ of PRP, topically applied on the treated area. The face was cleaned with normal saline at the end, and ice compresses were applied. In both the groups, the patients were advised strict photoprotection, and oral antibiotics, if necessary. They were reviewed after 1 week for any side effects and subsequently followed up at 1 month. 


\subsection{Clinical Assessment}

The degree of improvement was evaluated by Goodman and Baron's qualitative acne scarring grading system, Goodman and Baron's quantitative acne scar grading system [13], physician's global assessment (PGA) and visual analogue scale were used in both the groups. Clinical photographs were taken in each visit. All the patients were examined for side effects such as hypo- or hyperpigmentation, persistent erythema, oedema.

\subsection{Statistical Analysis}

The data was coded and entered into Microsoft Excel spreadsheet. Analysis was done using Statistical Package for Social Service (SPSS version 20 IBM SPSS Statistics Inc., Chicago, Illinois, USA) Windows software program. Descriptive statistics included computation of percentages, means and standard deviations. The independent $t$ test (for quantitative data within two groups) and paired t test (for quantitative data to compare before and after observations) was used for quantitative data comparison of all clinical indicators. Chi-square test used for qualitative data whenever two or more than two groups were used to compare. Level of significance was set at $\mathrm{P} \leq 0.05$.

\section{Results}

Demographics of the patients included in the study are given in table 1. Both the groups were significantly indifferent at baseline with respect to age and sex distribution, duration of disease, baseline Goodman and Baron's qualitative acne scar grade and baseline Goodman and Baron's quantitative acne scar scores $(\mathrm{P}$-value $>0.05)$ hence, the two groups were comparable before starting the therapy.

The results of two groups were compared in terms of improvement in Goodman and Baron's qualitative acne scar grades, decrease in mean Goodman and Baron's quantitative acne scar scores, percentage decrease in mean Goodman and Baron's quantitative acne scar scores, physician global assessment and visual analogue scale. Before and after clinical photographs for one patient in each group are shown in figures 1, 2 (Microneedling) and 3, 4 (Microneedling with PRP).

Table 1. Demography of the 40 patients included in the study.

\begin{tabular}{llll}
\hline Demographic data & Group 1 & Group 2 & P value \\
\hline Age (Years) (Mean \pm SD) & $23.25 \pm 4.17$ & $25.15 \pm 4.01$ & 0.15 \\
Sex ratio (Male: Female) & $3: 1$ & $4: 1$ & 0.70 \\
Duration of disease in months (Mean \pm SD) & $39 \pm 14.1$ & $42 \pm 17.19$ & 0.55 \\
Baseline Goodman and Baron's qualitative acne scar grade according to percentage (Grade 3 and Grade 4) & $15 \%, 85 \%$ & $25 \%, 75 \%$ & 0.42 \\
Baseline Goodman and Baron's quantitative acne scar scores Mean \pm SD & $13.8 \pm 3.75$ & $13.1 \pm 3.16$ & 0.52 \\
\hline
\end{tabular}

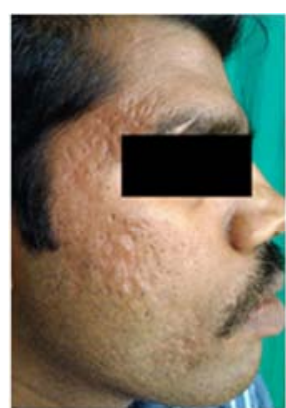

(a)

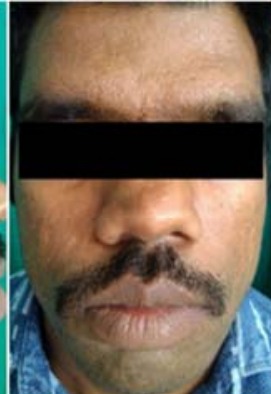

(b)

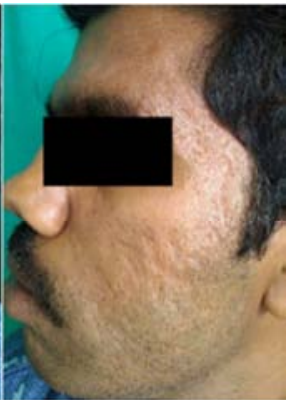

(c)

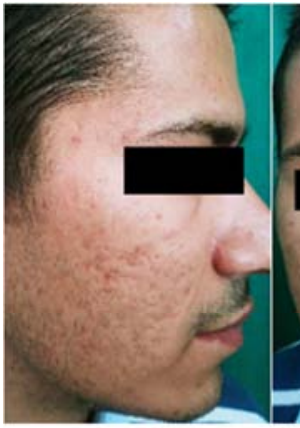

(a)

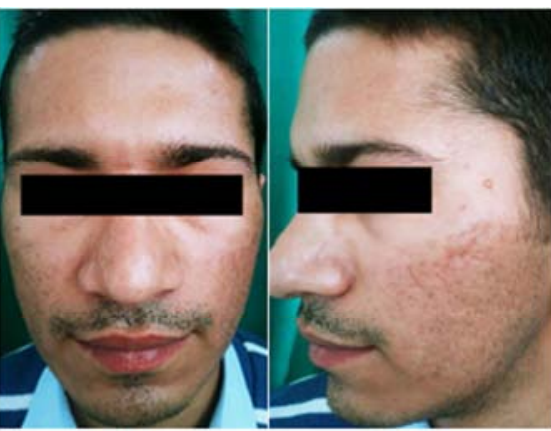

(b) (c)

Figure 1. At baseline (Group 1)

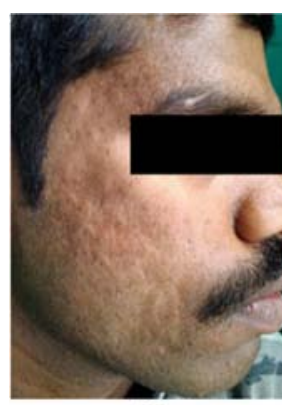

(a)

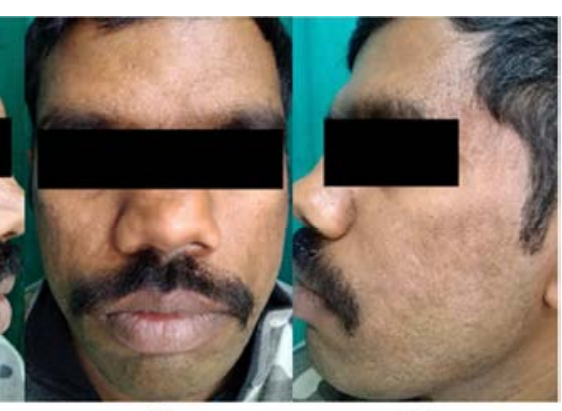

(b) (c)

Figure 2. At the end of 1 month after therapy (Group 1).

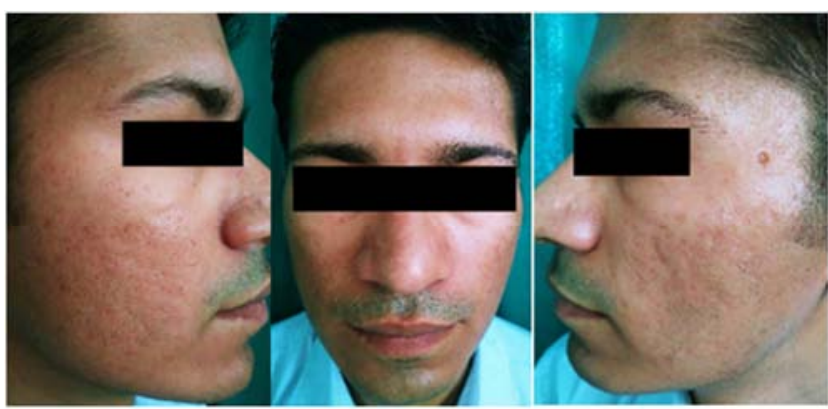

(b)

(c)

Figure 4. At the end of 1 month after therapy (Group 2).

On calculating Goodman and Baron's qualitative scores, a 
statistically significant difference was noted between the two groups at the end of 16 weeks $(P$ value $<0.001)$ as shown in Table 2. There is a significant improvement in term of Goodman and Baron's quantitative acne scar scores noted at the end of therapy between the two groups $(\mathrm{P}$ value $<0.001)$ as shown in Table 3.

It was also observed that there was a significant improvement in terms of mean percentage reduction in Goodman and Baron's quantitative acne scar scores of the two groups at the end of the therapy as shown in table 4, figure 5. On physician global assessment more percentage of patients belonging to group 2 who reported giving excellent $(15 \%)$, good $(25 \%)$, fair $(50 \%)$ and poor response $(10 \%)$ as compare to group 1 where patients gave good $(10 \%)$, fair $(60 \%)$ and poor $(30 \%)$ and none gave excellent response $(0 \%)$, which was statistically significant at the end of treatment (P value 0.03). Figure 6.

On visual analogue score, there was greater improvement in Group 2 after the treatment indicating greater patient satisfaction in the group treated with the combination therapy (P value-0.02). Figure 7 .

With respect to side effects, both the procedures were well tolerated by patients and no major side effects were observed in either of the groups. In both the groups, 2 out of 20 patients developed post inflammatory hyperpigmentation. All patients in both groups showed transient oedema after the procedure which lasted for 3 days and subsided on its own without treatment. None of the patients in the two groups showed, persistent erythema, oedema or post inflammatory hypopigmentation. Figure 8.

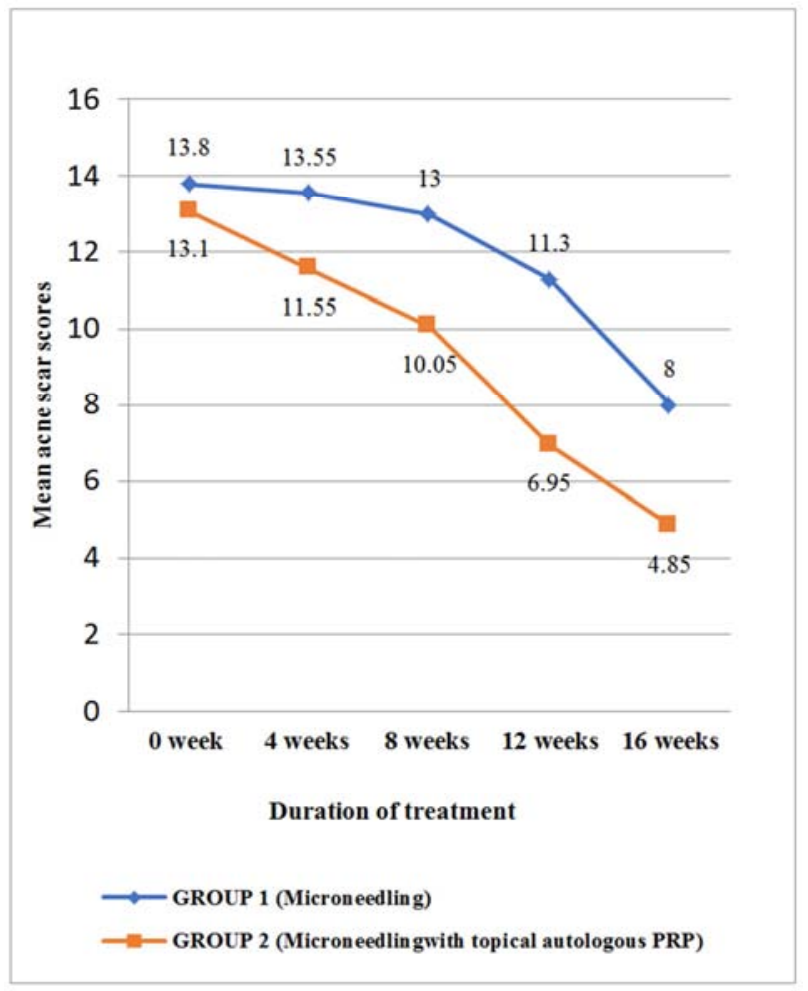

Figure 5. Mean percentage reduction in Goodman and Baron's quantitative acne scar scores in group 1 (Microneedling) and Group 2 (Microneedling with topical autologous PRP).

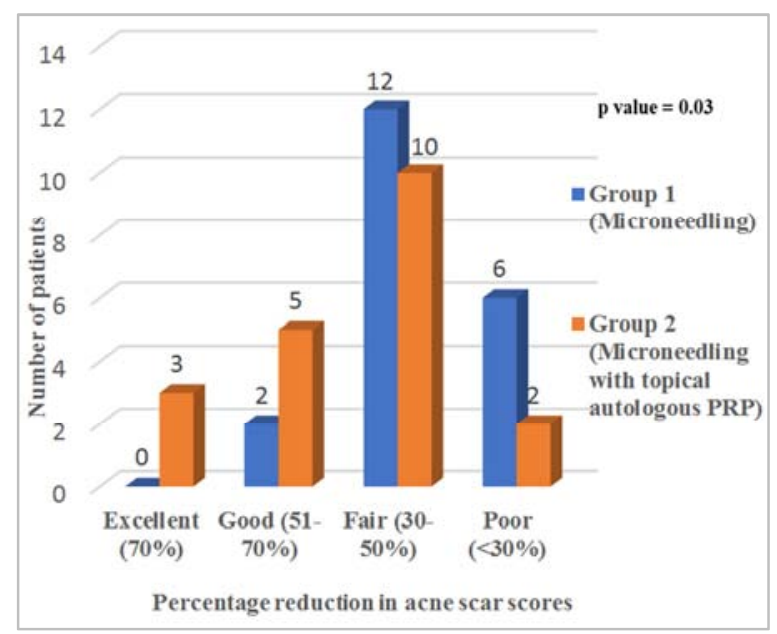

Figure 6. Physician's global assessment.

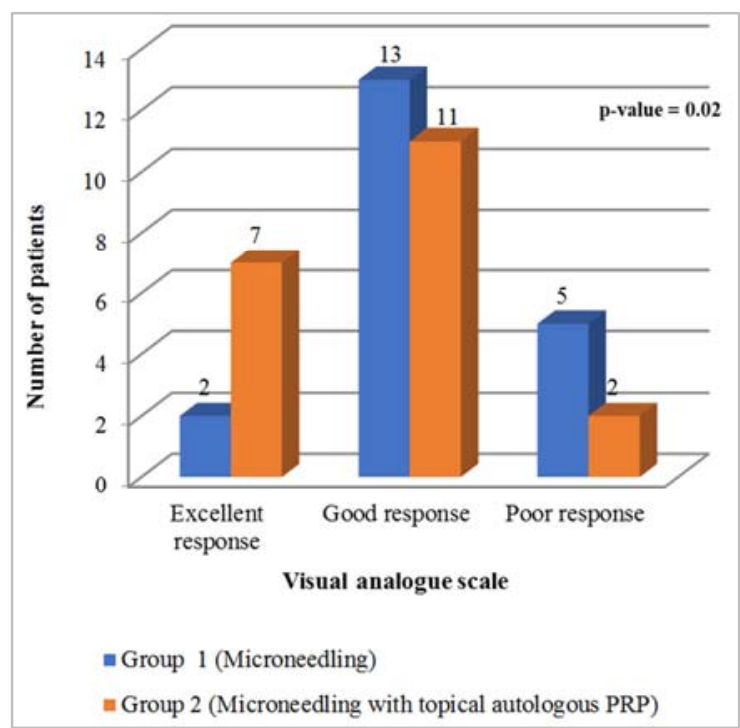

Figure 7. Visual analogue scale.

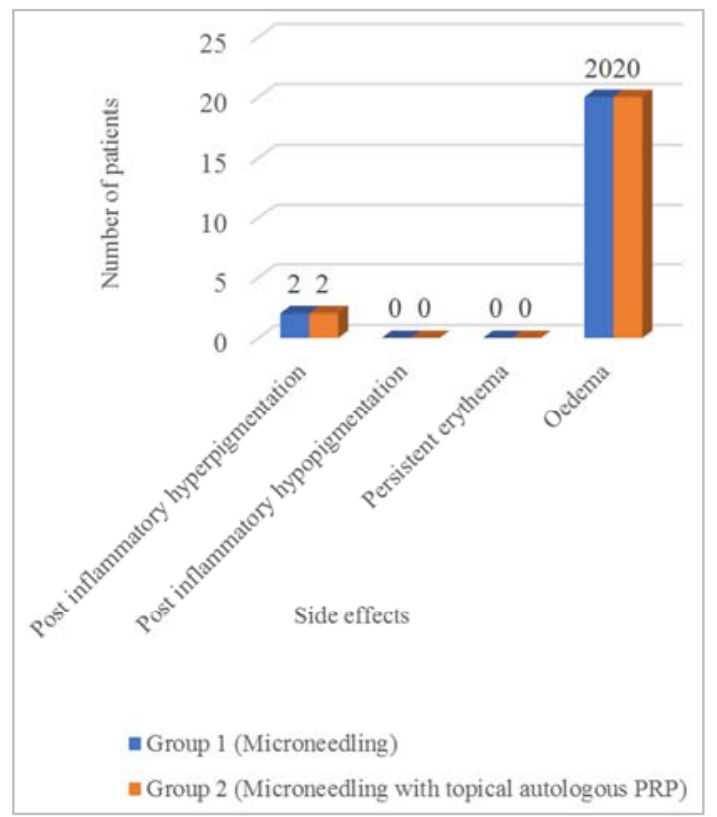

Figure 8. Side effects. 
Table 2. Distribution of cases according to Goodman and Baron's qualitative acne scar grading system in the two group at baseline and at the end of 16 weeks therapy.

\begin{tabular}{|c|c|c|c|c|c|c|c|c|}
\hline \multirow{3}{*}{$\begin{array}{l}\text { Acne scar } \\
\text { grade }\end{array}$} & \multicolumn{4}{|c|}{ At baseline } & \multicolumn{4}{|c|}{ At the end of therapy } \\
\hline & \multicolumn{2}{|l|}{ Group 1} & \multicolumn{2}{|l|}{ Group 2} & \multicolumn{2}{|l|}{ Group 1} & \multicolumn{2}{|l|}{ Group 2} \\
\hline & No of Pts & $\%$ of cases & No of pts & $\%$ of cases & No of pts & $\%$ of cases & No of pts & $\%$ of cases \\
\hline Grade 1 & 0 & 0 & 0 & 0 & 0 & 0 & 0 & 0 \\
\hline Grade 2 & 0 & 0 & 0 & 0 & 4 & 20 & 13 & 65 \\
\hline Grade 3 & 3 & 15 & 5 & 25 & 7 & 35 & 7 & 35 \\
\hline Grade 4 & 17 & 85 & 15 & 75 & 9 & 45 & 0 & 0 \\
\hline $\mathrm{P}$ value & 0.42 & & & & $0.001(\mathrm{~S})$ & & & \\
\hline
\end{tabular}

Table 3. Mean Goodman and Baron's quantitative acne scar scores at baseline and at the end of 16 weeks of therapy.

\begin{tabular}{llll}
\hline Mean acne score $($ Mean \pm SD) & Group 1 (Mean \pm SD) & Group 2 (Mean \pm SD) & P value \\
\hline At baseline & $13.8 \pm 3.75$ & $13.1 \pm 3.16$ & 0.52 \\
At 1 month after the fourth session & $8 \pm 2.61$ & $4.85 \pm 0.98$ & $0.001(\mathrm{~S})$ \\
\hline
\end{tabular}

Table 4. Assessment of improvement in terms of mean percentage reduction in Goodman and Baron's quantitative acne scar scores.

\begin{tabular}{llll}
\hline Mean percentage reduction in quantitative acne scar scores & Group 1 & Group 2 & P value \\
\hline Mean \pm SD & $42.33 \pm 8.89 \%$ & $62.06 \pm 6.15 \%$ & $0.001(\mathrm{~S})$ \\
\hline
\end{tabular}

\section{Discussion}

Atrophic acne scarring which can occur as a complication of acne vulgaris, can be associated with mental and social distress [14]. Many treatments are being offered for treating atrophic scars such as chemical peeling, subcision, dermabrasion, microneedling, fillers, ablative and nonablative lasers each of which has their own limitation [15]. Over the last few years, more newer treatments are being added to overcome these limitations. Microneedling therapy also known as collagen induction therapy and platelet rich plasma therapy are newer worthwhile options $[11,16]$. Microneedling done by using a dermaroller, is an effective procedure with minimal side effects [17]. PRP acts as rich source of autologous growth factors such as epidermal growth factor, platelet-derived growth factor, transforming growth factor beta and vascular endothelial growth factor both synergistically work together with growth factors produced by microneedling to augment the wound-healing response [17]. An earlier study done by Porwal S et al which was a comparative study comparing combination of dermaroller and intralesional platelet rich plasma versus dermaroller alone in acne scars and assessment of quality of life before and after treatment. The study showed a statistically significant difference between the two groups after the treatment with a higher percentage reduction of Goodman and Baron's quantitative acne scar scores in the combination group [17]. In a split face study by Fabbrocini et al, severity score analysis showed that acne scars treated with skin needling in combination with topical PRP application, had higher improvement than skin needling alone [11]. Ibrahim MK et al studied 35 patients with acne scars and reported that both microneedling and microneedling with topical PRP showed good results with less healing time observed in the combination group [18]. A recent systemic review by Long $\mathrm{T}$ et at also concluded that microneedling or subcision with PRP was more efficacious over microneedling or subcision alone [19].

In our study male outnumbered females (31:9); which is in agreement with a study done by Chawla $\mathrm{S}$ in which also male patients $(70 \%)$ outnumbered female patients [10]. Mean age of patients years in group 1 was $23.25 \pm 4.17$ and in group 2 was $25.15 \pm 4.01$ which was statistically not significant and maximum numbers were between the age group of 18-23 years and 24-29 years of age. Majid also observed age of the patients ranged from 13 to 34 years, with the mean age of 22.4 years [20].

Significant improvement in Goodman and Baron's qualitative acne scar grades were observed early in group 2 as compared to group 1. Our observation is in concordance with the findings of a recent study done by Yaseen $U$ et al [15] who had studied the efficacy of combination of platelet rich plasma (PRP) and microneedling in the management of atrophic acne scars and have also reported significant improvement in Goodman and Baron's qualitative acne scar grades with combination of platelet rich plasma and microneedling [15]. Statistically significant difference in mean Goodman and Baron's quantitative acne scar score between the two groups was observed after 8 weeks of therapy which continued to be statistically highly significant till the end of 16 weeks of therapy. The difference in mean percentage reduction in Goodman and Baron's quantitative acne scar scores between the two groups was statistically highly significant ( $\mathrm{p}$ value $=0.001$ ) at the end of 16 weeks of therapy. Thus, it was observed that the mean percentage reduction in Goodman and Baron's quantitative acne scar scores of group 2 was significantly higher than group 1 indicating that combination therapy was significantly better. Our study is in concordance with the study done by Porwal S et al [16]. Combination group showed better response on physician global assessment with a significant difference $(p$ value $=0.03$ ). On analyzing patient's assessment using visual analogue scale, it was found that in group 2 patient gave a 
better response to the treatment than group 1. Our finding is also in concordance with the study done by Porwal $\mathrm{S}$ et al who have also reported greater improvement in visual analogue score in group treated with combination therapy [16].

The side effects noted were few and there was not much difference between the two groups in occurrence of side effects. Erythema and oedema occurred after the procedure and persisted for 3-4 days and subsides without treatment. The side effect profile of our study is similar to the study by Porwal $\mathrm{S}$ et al, Fabbrocini $\mathrm{G}$ et al and Yaseen $\mathrm{U}$ et al who had also reported post-dermaroller transient erythema and oedema lasting for 1-4 days in both the groups $[16,11,15]$.

In a resource poor country like India where advance and expensive technology cannot be available everywhere, microneedling with topical autologous PRP can offer as a better advantage in the treatment of acne scars as it is relatively cheap, safe with few side effects in addition it requires less time and less painful that intradermal PRP.

\section{Conclusion}

We concluded that microneedling using dermaroller in combination with topical autologous platelet rich plasma is more efficacious than microneedling using dermaroller alone in treating atrophic acne scars. Both the procedures are safe and well tolerated by the patients.

\section{Ethics Statement}

Prior written consent of both the patients being photographed have been taken before submitting for publication.

\section{References}

[1] Fabbrocini F, Annunziatia MC, Monfrecolo G. Acne scars: Pathogenesis, Classification and treatment. Dermatol Res Prac. 2010. doi: $10.1155 / 2010 / 893080$.

[2] Lauermann FT, Duquia RP, Almeida HL, de Souza PRM, Breunig JDA. Acne scars in 18 year old male adolescents: a population based study of prevalence and associated factors. An Bras Dermatol 2016; 91: 291-5.

[3] El-Domyati M, Barakat M, Awad S, Medhat W, El-Fakahany H, Farag H. Microneedling Therapy for Atrophic Acne Scars: An Objective Evaluation. J Clin Aesthet Dermatol 2015; 8: $36-42$

[4] Jacob CI, Dover JS, Kaminer MS. Acne scarring: a classification system and review of treatment options 2001; 19: 109-17.

[5] Connoly D, Vu HL, Mariwalla K, Saedi N. Acne Scarring:
Pathogenesis, Evaluation and Treatment Options. J Clin Aesthet Dermatol 2017; 10: 12-23.

[6] Doddaballapur S. Microneedling with Dermaroller. J Cutan Aesthet Surg 2009; 2: 110-11.

[7] Singh A, Yadav S. Microneedling: Advances and Widening Horizons. Indian Dermatology Online Journal 2016; 7: 244-47.

[8] Leo MS, Kumar AS, Kirit R, konathan R, Sivamani RK. Systematic review of the use of platelet-rich plasma in aesthetic dermatology. J Cosmet Dermatol 2015; 14: 315-23.

[9] Saeed M, Elethawi A, Al-Ani Z, Abdullah R. Application of fractional microneedling radio-frequency and autologous platelet in managing facial Acne scars. Br J Med Med Res 2016; 14: 1-18.

[10] Chawla S. Split face comparative study of microneedling with PRP versus microneedling with vitamin $\mathrm{C}$ in treating atrophic post acne scars. J Cutan Aesthet Surg 2014; 7: 209-12.

[11] Fabbrocini G, De Vita V, Pastore F, Panariello L, Fardella N, Sepulveres R, et al. Combined use of skin needling and platelet-rich plasma in acne scarring treatment. Cosmet Dermatol 2011; 24: 177-83.

[12] Goodman GJ, Baron JA. Postacne scarring: A qualitative global scarring grading system. Dermatol Surg 2006; 32 (12): 1458-66.

[13] Goodman GJ, Baron JA. Postacne scarring: A quantitative global scarring grading system. J Cosmet Dermatol 2006; 5: 48-52.

[14] Cotterill JA, Cunliffe WJ. Suicide in dermatologic patients. $\mathrm{Br}$ J Dermatol 1997; 137: 246-50.

[15] Ibrahim ZA, El-Ashmawy AA, Shora OA. Therapeutic effect of microneedling and autologous platelet rich plasma in the treatment of atrophic scars: A randomized study. J Cosmet Dermatol 2017; 1-12.

[16] Yaseen U, Shah S, Bashir A. Combination of platelet rich plasma and microneedling in the management of atrophic acne scars. Int J Res Dermatol 2017; 3 (3): 346-50.

[17] Porwal S, Chahar YS, Singh PK. A comparative study of combined dermaroller and platelet-rich plasma versus dermaroller alone in acne scars and assessment of quality of life before and after treatment. Indian J Dermatol 2018; 63: 403-8.

[18] Ibrahim MK, Ibrahim SM, Salem AM. Skin microneedling plus platelet-rich plasma versus skin microneedling alone in the treatment of atrophic post acne scars: a split face comparative study. J Dermatolog Treat 2018; 63: 403-8.

[19] Long T, Gupta A, Ma S, Hsu S. Platelet-rich plasma in noninvasive procedures for atrophic acne scars: A systematic review and meta-analysis. J Cosmet Dermatol. 2020; 00: 1-9. https://doi.org/10.1111/jocd.13331.

[20] Majid I. Microneedling Therapy in Atrophic Facial Scars: An Objective Assessment. J Cutan Aesthet Surg 2009; 2: 26-3. 